\title{
AVERAGE NUMBER OF LOCAL MINIMA FOR THREE-DIMENSIONAL INTEGRAL LATTICES
}

\author{
A. A. ILLARIONOV
}

Abstract. An asymptotic formula is found for the average number of local minima of three-dimensional complete integral lattices with determinant in the interval $[1, N]$. This is a generalization to the two-dimensional case of the classical result about the average length of a finite continued fraction with denominator belonging to $[1, N]$.

\section{Notation}

$\mathbb{R}_{+}$is the set of positive real numbers;

$X^{s \times s}$ is the set of $(s \times s)$-matrices with entries in $X$;

$\mathrm{GL}_{s}(\mathbb{R})$ is the set of nonsingular matrices in $\mathbb{R}^{s \times s}$;

$\mathrm{GL}_{s}(X ; N)=\left\{M \in X^{s \times s}: \operatorname{det} M=N\right\}$;

for $m^{(1)}, \ldots, m^{(s)} \in \mathbb{R}^{s}$, the symbol $\left[m^{(1)}, \ldots, m^{(s)}\right]$ denotes the matrix with the columns $m^{(1)^{T}}, \ldots, m^{(s)^{T}}$;

$\partial \Omega$ is the boundary of a set $\Omega$, and $\bar{\Omega}$ is the closure of $\Omega$;

$\# X$ is the cardinality of a finite set $X$.

We write $f(x) \ll g(x)$ (or $f(x)=O(g(x))$ ) for $x \in X$ if there exists a constant $C>0$ such that $|f(x)| \leq C \cdot g(x)$ for all $x \in X$. If $C$ depends on a parameter $\theta$, we write $f(x) \ll_{\theta} g(x)\left(\right.$ or $\left.f(x)=O_{\theta}(g(x))\right)$. We write $f \asymp g$ if $f \ll g \ll f$.

\section{INTRODUCTION}

Two generalizations of continued fractions to the multidimensional case emerged at the end of the 19th century. One of them was due to F. Klein [1, the other was suggested by Voronol [2, and, independently, by Minkowski [3]. Each one is related to isolation and the study of certain sets of nodes of an $s$-dimensional lattice $\Gamma$, specifically, the Klein polytops and (accordingly) the set $\mathfrak{M}(\Gamma)$ of all local minima for $\Gamma$.

We recall the definitions. A complete s-dimensional integral lattice is a set of the form

$$
\Gamma=\left\{\sum_{i=1}^{s} k_{i} m^{(i)}: k_{i} \in \mathbb{Z}\right\},
$$

where the $m^{(i)}(i=1, \ldots, s)$ are linearly independent vectors in $\mathbb{Z}^{s}$ (a basis of $\Gamma$ ).

The quantity $\operatorname{det} \Gamma=\left|\operatorname{det}\left(\left(m_{j}^{(i)}\right)\right)\right|$ is called the determinant of $\Gamma$.

A nonzero node $\gamma \in \Gamma$ is called a local minimum of $\Gamma$ if there is no other nonzero node $\gamma^{\prime} \in \Gamma$ with

$$
\left|\gamma_{i}^{\prime}\right| \leq\left|\gamma_{i}\right| \quad i=1, \ldots, s, \quad \sum_{i=1}^{s}\left|\gamma_{i}^{\prime}\right|<\sum_{i=1}^{s}\left|\gamma_{i}^{\prime}\right| .
$$

2010 Mathematics Subject Classification. Primary 11H06.

Key words and phrases. Lattice, local minimum, multidimensional continued fraction, Euclid algorithm.

Supported by RFBR (grants nos. 10-01-98002r-siberia-a , 11-01-00628-a), by FED RAS (grants nos. 11-III-V-01M-002, 09-I-114-03), and by the grant MD-2339.2010.1 of the President of RF. 
We use the following notation:

$\mathcal{L}_{s}(\mathbb{Z} ; N)$ is the set of all complete $s$-dimensional integral lattices;

$\mathfrak{M}(\Gamma)$ is the set of local minima of a lattice $\Gamma$.

The construction of Voronol and Minkowski was motivated by the Lagrange theorem on best approximation with the help of continued fractions. For instance, if $\alpha \in(0,1 / 2)$, then for the lattice $\Gamma_{\alpha}$ with the basis $(1, \alpha),(0,1)$ we have

$$
\mathfrak{M}\left(\Gamma_{\alpha}\right)=\left\{ \pm\left(Q_{i}, \alpha Q_{i}-P_{i}\right): i=0,1, \ldots\right\},
$$

where $Q_{0}=0, P_{0}=1$, and $P_{i} / Q_{i}$ is the $i$ th convergent for $\alpha$ if $i \geq 1$.

The notion of a local minimum emerges in various mathematical fields. For example, it was observed in [4, 5, that the set $\mathfrak{M}(\Gamma)$ of local minima determines the discrepancy of Korobov's multidimensional quadrature formulas (see also [6]).

Despite a considerable interest, fairly little is known about the number of local minima for lattices in dimensions 3 and higher (for two-dimensional lattices, the number of local minima is determined by the length of the continued fraction expansion for the corresponding $\alpha$ ). Only the following estimates (from above and from below) are known:

$$
\begin{gathered}
\# \mathfrak{M}(\Gamma) \ll_{s} \ln ^{s-1} N \quad \forall \Gamma \in \mathcal{L}\left(\mathbb{Z}^{s} ; N\right), \quad N \geq 2, \\
\sum_{\Gamma \in \mathcal{L}\left(\mathbb{Z}^{s} ; N\right)} \# \mathfrak{M}(\Gamma) \gg_{s} N^{s-1} \ln ^{s-1} N .
\end{gathered}
$$

For complete lattices, inequality (2) was proved in [4, 5] (see 7] for an estimate of the constant), and inequality (3) was proved in [10]. The results of [10] show also that (2) is sharp, up to a constant depending on the dimension $s$. Similar estimates for noncomplete lattices were obtained in 8 .

We introduce the quantity

$$
E_{s}(N)=\frac{\sum_{n=1}^{N} \sum_{\Gamma \in \mathcal{L}_{s}(\mathbb{Z} ; n)} \# \mathfrak{M}(\Gamma)}{\sum_{n=1}^{N} \# \mathcal{L}_{s}(\mathbb{Z} ; n)},
$$

i.e., the average number or local minima for $s$-dimensional complete integral lattices with determinant belonging to $[1, N]$.

It can easily be proved (see Lemma 2 below) that

$$
\sum_{n=1}^{N} \# \mathcal{L}_{s}(\mathbb{Z} ; n) \ll_{s} N^{s} .
$$

Using (2), (3), and (4), it is easy to observe that

$$
E_{s}(N) \asymp_{s} \ln ^{s-1} N \text { for } \quad N \geq 2 .
$$

Conjecture. There exists a constant $C(s)$ such that

$$
E_{s}(N) \sim C(s) \ln ^{s-1} N \quad \text { as } \quad N \rightarrow+\infty .
$$

For $s=2$, the asymptotic formula (5) can easily be deduced from Heilbronn's classical result [9] about the average length of a finite continued fraction (see Lemma 3 below); moreover,

$$
C(2)=\frac{4 \ln 2}{\zeta(2)}
$$

Throughout, $\zeta$ stands for the Riemann zeta-function.

Our aim in the present paper is to prove formula (5) for $s=3$. This generalizes classical results about the average length of a finite continued fraction with denominator in $[1, N]$ (see [11, 9]) to the two-dimensional case.

The author is grateful to V. A. Bykovskiu for attention to this work and useful advice. 


\section{$\S 1$. Statement of the main Result}

If

$$
\Omega=\left\{\left(\begin{array}{lll}
a_{1} & b_{1} & c_{1} \\
a_{2} & b_{2} & c_{2} \\
a_{3} & b_{3} & c_{3}
\end{array}\right) \in \mathrm{GL}_{3}(\mathbb{R}): \begin{array}{l}
\left(b_{1}, c_{1}\right) \in W_{1} \cdot a_{1} \\
\left(a_{2}, c_{2}\right) \in W_{2} \cdot b_{2} \\
\left(a_{3}, b_{3}\right) \in W_{3} \cdot c_{3},
\end{array}\right\}
$$

where the $W_{i}$ are Jordan measurable subsets of $\mathbb{R}^{2}$, then

$$
\mu(\Omega)=\int_{W_{1} \times W_{2} \times W_{3}} \frac{d b_{1} d c_{1} d a_{2} d c_{2} d a_{3} d b_{3}}{|\operatorname{det} M(a, b, c)|^{3}}, \quad M(a, b, c)=\left(\begin{array}{ccc}
1 & b_{1} & c_{1} \\
a_{2} & 1 & c_{2} \\
a_{3} & b_{3} & 1
\end{array}\right) .
$$

Note that $\mu$ is invariant under the action of the group $\mathrm{GL}_{3}(\mathbb{R})$.

We introduce the following sets of matrices:

$$
\begin{aligned}
& \Omega_{1}=\left\{\left(\begin{array}{ccc}
a_{1} & -b_{1} & -c_{1} \\
a_{2} & b_{2} & -c_{2} \\
a_{3} & -b_{3} & c_{3}
\end{array}\right): a_{1}<b_{1}+c_{1}\right\}, \\
& \Omega_{2}=\left\{\left(\begin{array}{ccc}
a_{1} & -b_{1} & -c_{1} \\
a_{2} & b_{2} & c_{2} \\
a_{3} & -b_{3} & c_{3}
\end{array}\right): \begin{array}{l}
a_{2}>c_{2} \\
\text { or } \\
c_{1}>b_{1}
\end{array}\right\}, \\
& \Omega_{3}=\left\{\left(\begin{array}{ccc}
a_{1} & -b_{1} & -c_{1} \\
a_{2} & b_{2} & -c_{2} \\
a_{3} & b_{3} & c_{3}
\end{array}\right)\right\}, \\
& \Omega_{4}=\left\{\left(\begin{array}{ccc}
a_{1} & -b_{1} & c_{1} \\
a_{2} & b_{2} & -c_{2} \\
a_{3} & b_{3} & c_{3}
\end{array}\right): \begin{array}{l}
b_{2}<a_{2}+c_{2}>c_{1}
\end{array}\right\},
\end{aligned}
$$

where

$$
\begin{aligned}
& a_{i}, b_{i}, c_{i} \in \mathbb{R}_{+}, \quad i=1,2,3, \\
& a_{1}>b_{1}, c_{1}, \quad b_{2}>a_{2}, c_{2}, \quad c_{3}>a_{3}>b_{3} .
\end{aligned}
$$

We state the main result of this paper.

Theorem 1. For $N$ running through the natural numbers, we have

$$
E_{3}(N)=\frac{4 C}{\zeta(2) \zeta(3)} \ln ^{2} N+O(\ln N+1)
$$

where $C=\mu\left(\Omega_{1}\right)+\mu\left(\Omega_{2}\right)+2 \mu\left(\Omega_{3}\right)+2 \mu\left(\Omega_{4}\right)$.

Remark 1. Apparently, $C$ cannot be expressed in terms of known constants. The calculation of the quantities $\mu\left(\Omega_{i}\right)$ reduces to evaluating certain 6 -fold integrals, in which two integrations can be done explicitly. Approximately, we have

$$
\begin{gathered}
\mu\left(\Omega_{1}\right) \approx 0.0442, \quad \mu\left(\Omega_{2}\right) \approx 0.0922, \quad \mu\left(\Omega_{3}\right) \approx 0.1119, \quad \mu\left(\Omega_{4}\right) \approx 0.0268 \\
C \approx 0.4120, \quad C(3)=\frac{4 C}{\zeta(2) \zeta(3)} \approx 0.8335
\end{gathered}
$$

Note that $C(2) \approx 1.6855$.

The next section contains some preliminaries. In $\S 3$ it is shown that finding $E_{3}(N)$ reduces to the calculation of the number of integral matrices of a specific form. In $\S 4$, a formula is presented for the number of integral matrices in a given domain. In the last section we finish the proof of Theorem 1 . 


\section{$\S 2$. Preliminary information}

A matrix is said to be basic if its columns constitute a basis of the lattice $\Gamma$.

Lemma 1. For every lattice $\Gamma \in \mathcal{L}_{s}(\mathbb{Z} ; n)$, there exists a unique basic matrix of the form

$$
M=\left(\begin{array}{ccccc}
m_{1} & m_{12} & m_{13} & \ldots & m_{1 s} \\
0 & m_{2} & m_{23} & \ldots & m_{2 s} \\
0 & 0 & m_{3} & \ldots & m_{2 s} \\
\vdots & \vdots & \vdots & \ddots & \vdots \\
0 & 0 & 0 & \ldots & m_{s}
\end{array}\right)
$$

where $0<m_{i j} \leq m_{i}, i=i+1, \ldots, s, j=1, \ldots, s, m_{1} m_{2} \cdots m_{s}=n$.

Proof. The existence is well known (see, e.g., [17, Chapter 1]). We prove uniqueness. Suppose $M$ and $M^{\prime}$ are two matrices of this sort. Then there is a unimodular integral matrix $S$ with $M=M^{\prime} \cdot S$, and it can easily be proved consecutively that

$$
\begin{aligned}
s_{i j}=0 \text { for } & i>j \Longrightarrow s_{i i}=1 \Longrightarrow m_{i}=m_{i}^{\prime}, i=1, \ldots, s \\
& \Longrightarrow m_{i j} \equiv m_{i j}^{\prime} \quad\left(\bmod m_{i}\right) \forall j \neq i \Longrightarrow m_{i j}=m_{i j}^{\prime}, i, j=1, \ldots, s .
\end{aligned}
$$

Lemma 2. For $s \geq 2$ and $N \in \mathbb{N}$, we have

$$
\sum_{n=1}^{N} \# \mathcal{L}_{s}(\mathbb{Z} ; n)=\frac{\zeta(2) \zeta(3) \cdots \zeta(s)}{s} N^{s}+O_{s}\left(N^{s-1} \ln N+1\right)
$$

Proof. By Lemma 1 the number of lattices in $\mathcal{L}_{s}(\mathbb{Z} ; n)$ is equal to the number of integral matrices of the form (6). Therefore,

$$
\begin{gathered}
\# \mathcal{L}_{s}(\mathbb{Z} ; n)=\sum_{\substack{m_{1}, \ldots, m_{s} \in \mathbb{N}, m_{1} \cdots \cdots m_{s}=n}} m_{1}^{s-1} m_{2}^{s-2} \cdots m_{s-1}, \\
\sum_{n=1}^{N} \# \mathcal{L}_{s}(\mathbb{Z} ; n)=\sum_{\substack{m_{1}, \ldots, m_{s} \in \mathbb{N}, m_{1} \cdots m_{s} \leq N}} m_{1}^{s-1} m_{2}^{s-2} \cdots m_{s-1} \\
=\frac{N^{s}}{s} \sum_{\substack{m_{2}, \ldots, m_{s} \in \mathbb{N}, m_{2} \cdots m_{s} \leq N}} \frac{1}{m_{2}^{2} m_{3}^{3} \cdots m_{s}^{s}}+O_{s}\left(\sum_{\substack{m_{2}, \ldots, m_{s} \in \mathbb{N}, m_{2} \cdots m_{s} \leq N}} \frac{N^{s-1}}{m_{2} m_{3}^{2} \cdots m_{s}^{s-1}}\right) \\
=\frac{N^{s}}{s} \zeta(2) \zeta(3) \cdots \zeta(s)+O_{s}\left(N^{s-1} \ln N+1\right) .
\end{gathered}
$$

For completeness, we show how to deduce the asymptotic expression for the number of relative minima of two-dimensional lattices (though this result will not be used in the sequel). We put

$$
\sigma(N)=\sum_{d \mid N} d, \quad \Lambda(N)= \begin{cases}\ln p & \text { if } N=p^{k}, \quad p \text { is prime } \\ 0 & \text { otherwise }\end{cases}
$$

these are the sum of divisors of $N$ and the Mangoldt function. 
Lemma 3. For every $N \geq 2$, we have

$$
\begin{aligned}
\sum_{\Gamma \in \mathcal{L}_{2}(\mathbb{Z} ; N)} \# \mathfrak{M}(\Gamma) & =\frac{4 \ln 2}{\zeta(2)} \sum_{d \backslash N} d\left(\ln d-\sum_{r \backslash d} \frac{\Lambda(r)}{r}\right)+O(\sigma(N)), \\
\sum_{n=1}^{N} \sum_{\Gamma \in \mathcal{L}_{2}(\mathbb{Z} ; n)} \# \mathfrak{M}(\Gamma) & =2 \ln (2) \cdot N^{2} \ln N+O\left(N^{2}\right), \\
E_{2}(N) & =\frac{4 \ln 2}{\zeta(2)} \ln N+O(1) .
\end{aligned}
$$

Proof. We denote by $l_{d}(a)$ the length of the continued fraction expansion of $\frac{a}{d}$, and by $\Gamma(a, d)$ the lattice with the basis $(d, 0),(a, 1)(a, d \in \mathbb{N})$. Formula (1) shows that

$$
\# \mathfrak{M}(\Gamma(a, d))=2 l_{d}(a)+2 \text { for } 0<a<d, \quad a \neq \frac{d}{2} .
$$

Since $\# \mathfrak{M}(\Gamma(a, d))=O(1)$ and $l_{d}(a)=O(1)$ for $d \in\{a, 2 a\}$, we obtain

$$
\sum_{a=1}^{d} \# \mathfrak{M}(\Gamma(a, d))=2 \sum_{a=1}^{d} l_{d}(a)+O(d) .
$$

A well-known result by Porter [12] implies the following formula (see [13, $§$ 4.5.3]):

$$
\sum_{a=1}^{d} l_{d}(a)=\frac{2 \ln 2}{\zeta(2)} d\left(\ln d-\sum_{r \backslash d} \frac{\Lambda(r)}{r}\right)+O(d) .
$$

By Lemma 1 any lattice in $\mathcal{L}_{2}(\mathbb{Z} ; N)$ has a unique basis of the form

$$
(d, 0), \quad\left(a, d^{\prime}\right),
$$

where $d d^{\prime}=N, 1 \leq a \leq d$. The transformation of nodes given by $\left(\gamma_{1}, \gamma_{2}\right) \rightarrow\left(\gamma_{1}, \gamma_{2} / d^{\prime}\right)$ transforms the lattice with the basis (13) to the lattice $\Gamma(a, d)$. Consequently,

$$
\sum_{\Gamma \in \mathcal{L}_{2}(\mathbb{Z} ; N)} \# \mathfrak{M}(\Gamma)=\sum_{d \backslash N} \sum_{a=1}^{d} \# \mathfrak{M}(\Gamma(a, d)) .
$$

Applying (11) and (12), we arrive at (8). Now, (9) follows from (8) by summation. It suffices to observe that

$$
\begin{aligned}
& \sum_{n=1}^{N} \sum_{d \backslash n} d \ln d=\sum_{n=1}^{N} \sum_{d \backslash n} \frac{n}{d} \cdot \ln (n / d)=\sum_{d=1}^{N} \sum_{\substack{d \leq n \leq N, n \equiv 0}} \frac{n}{d} \cdot \ln (n / d) \\
& =\sum_{d=1}^{N}\left(\frac{1}{d} \int_{d}^{N} \frac{t}{d} \cdot \ln (t / d) d t+O\left(\frac{N}{d} \ln (N / d)\right)\right) \\
& =\sum_{d=1}^{N}\left(\frac{N^{2}}{2 d^{2}} \ln (N / d)+O\left(\frac{N^{2}}{d^{2}}+\frac{N}{d} \ln (N / d)\right)\right)=\frac{N^{2} \ln N}{2} \zeta(2)+O\left(N^{2}\right), \\
& \sum_{n=1}^{N} \sum_{d \backslash n} d \sum_{r \backslash d} \frac{\Lambda(r)}{r}=\sum_{d=1}^{N} \sum_{r \backslash d} \sum_{\substack{1 \leq n \leq N, n \equiv 0}} d \cdot \frac{\Lambda(r)}{r} \ll N \sum_{d=1}^{N} \sum_{r \backslash d} \frac{\Lambda(r)}{r}=O\left(N^{2}\right), \\
& \sum_{n=1}^{N} \sigma(n)=O\left(N^{2}\right)
\end{aligned}
$$


This leads to (9). Formula (10) is a consequence of (9) and (7).

We pass to three-dimensional lattices. Put

$$
\begin{aligned}
\mathcal{U}(N) & =\left\{(\gamma, \Gamma): \Gamma \in \mathcal{L}_{3}(\mathbb{Z} ; N), \gamma \in \mathfrak{M}(\Gamma)\right\}, \quad N \in \mathbb{N}, \\
\mathcal{U}_{+}(N) & =\left\{(\gamma, \Gamma) \in \mathcal{U}(N): \gamma_{1,2,3}>0\right\} .
\end{aligned}
$$

By using (2), it is easy to show (see [8]) for more details) that, for every lattice in $\mathcal{L}_{3}(\mathbb{Z} ; n)$ with $n \geq 2$, the number of relative minima with at least one zero coordinate is $O(\ln n)$. So, (4) implies

$$
\sum_{\Gamma \in \mathcal{L}_{3}(\mathbb{Z} ; n)} \# \mathfrak{M}(\Gamma) \equiv \# \mathcal{U}(n)=2^{3} \cdot \# \mathcal{U}_{+}(n)+O\left(\ln n \cdot \# \mathcal{L}_{3}(\mathbb{Z} ; n)\right) .
$$

Taking (7) into account, we obtain

$$
E_{3}(N)=\frac{24}{N^{3} \zeta(2) \zeta(3)} \sum_{n=1}^{\infty} \mathcal{U}_{+}(n)+O(\ln N)
$$

For two-dimensional lattices, the calculation of the cardinality of the set

$$
\mathcal{U}_{+}^{(2)}(N)=\left\{(\gamma, \Gamma): \Gamma \in \mathcal{L}_{2}(\mathbb{Z} ; N), \quad \gamma \in \mathfrak{M}(\Gamma), \gamma_{1,2}>0\right\}
$$

is based on the following observation. Let $(\gamma, \Gamma) \in \mathcal{U}_{+}^{(2)}(N)$. We choose a node $b \in$ $\mathfrak{M}(\Gamma) \backslash\{a\}$ in accordance with the conditions

$$
\left|b_{1}\right| \leq a_{1}, \quad 0 \leq b_{2} \rightarrow \min .
$$

Then $b_{1} \leq 0, b_{2} \geq a_{2}$, and the vectors $a, b$ form a basis of $\Gamma$. Consequently, any pair $(a, \Gamma) \in \mathcal{U}_{+}^{(2)}(N)$ gives rise to a matrix

$$
A \in \omega(N)=\left\{\left(\begin{array}{cc}
a_{1} & -b_{1} \\
a_{2} & b_{2}
\end{array}\right) \in \mathrm{GL}_{2}(\mathbb{Z} ; N): 0 \leq b_{1} \leq a_{1}, \quad 0<a_{2} \leq b_{2}\right\} .
$$

The converse is also true. If $[a, b] \in \omega(N)$ and $\Gamma$ is the lattice with the basis $a, b$, then $a \in \mathfrak{M}(\Gamma)$ and (15) holds true. Therefore, the sets $\mathcal{U}_{+}^{(2)}(N)$ and $\omega(N)$ are in one-to-one correspondence. Consequently, \# $\mathcal{U}_{+}^{(2)}(N)=\# \omega(N)$.

For the first time, similar arguments were used in Heilbronn's classical paper 9 for calculation of the average length of a continued fraction. In the next section, we shall obtain a similar correspondence for three-dimensional lattices.

\section{§3. An analog of Heilbronn's Correspondence}

Along with the sets $\Omega_{i}(i=1,2,3,4)$ of matrices introduced above, we also employ the sets $\partial \Omega_{i}$ (the matrices belonging to the boundary of $\Omega_{i}$ ) and $\bar{\Omega}_{i}=\Omega_{i} \cup \partial \Omega_{i}$.

If $\Omega \subset \mathbb{R}^{3 \times 3}$, then $\omega(N)=\Omega \cap \mathrm{GL}_{3}(\mathbb{Z} ; N)$. In particular,

$$
\bar{\omega}_{i}(N)=\bar{\Omega}_{i} \cap \mathrm{GL}_{3}(\mathbb{Z} ; N), \quad \partial \omega_{i}(N)=\partial \Omega_{i} \cap \mathrm{GL}_{3}(\mathbb{Z} ; N) .
$$

Our aim in this section is the proof of the following lemma.

Lemma 4. For every natural number $N$, we have

$$
\begin{aligned}
\# \mathcal{U}_{+}(N)= & \# \omega_{1}(N)+\# \omega_{2}(N)+2 \cdot \# \omega_{3}(N)+2 \cdot \# \omega_{4}(N) \\
& +O\left(\sum_{i=1}^{4} \# \partial \omega_{i}(N)\right)+O_{\epsilon}\left(N^{1+\epsilon}\right) \quad \forall \epsilon>0
\end{aligned}
$$


This statement will be verified at the end of the section. The arguments are based on the considerations described below. Let $(a, \Gamma) \in \mathcal{U}_{+}(N)$. We choose nodes $b \in \mathfrak{M}(\Gamma) \backslash\{a\}$ and $c \in \mathfrak{M}(\Gamma) \backslash\{a, b\}$ in accordance with the following conditions:

$$
\begin{aligned}
& \left|b_{i}\right| \leq a_{i}, i=1,3, \quad 0 \leq b_{2} \rightarrow \min , \\
& \left|c_{1}\right| \leq a_{1}, \quad\left|c_{2}\right| \leq b_{2}, \quad 0 \leq c_{3} \rightarrow \min .
\end{aligned}
$$

If we discard certain "bad" cases (whose number is estimated by the remainder term in (16)) and eliminate certain "symmetric" variants (the sets $V_{2}(N)$ and $V_{3}(N)$ below), then $a, b, c$ is a basis of $\Gamma$, and moreover, the matrix $[a, b, c]$ belongs to the union of the sets $\omega_{i}(N)$.

We shall use the so-called minimal sets. Recall the definition.

Definition 1. A set $M \subset \Gamma \in \mathcal{L}_{s}(\mathbb{R})$ is said to be minimal if $M \subset \mathfrak{M}(\Gamma)$ and there is no nonzero node $\gamma \in \Gamma$ such that

$$
\left|\gamma_{i}\right|<\max _{\left(\eta_{1}, \ldots, \eta_{s}\right) \in M}\left|\eta_{i}\right|, \quad i=1, \ldots, s .
$$

The notion of a minimal set first appeared in the work of Voronor and of Minkowski (see [2, 14]) in connection with methods of constructing units in number fields. They analyzed various properties of minimal systems of two- and three-dimensional latices in general position (without proofs in the 3-dimensional case). A fairly exhaustive study of three-dimensional lattices in general position was done in [15. Minimal systems of lattices in $\mathcal{L}_{3}(\mathbb{Z})$ were investigated in [16].

In the present paper, we shall content ourselves with the following result. Denote by $\widetilde{\mathcal{L}}_{3}(\mathbb{Z} ; N)$ the set of lattices $\Gamma \in \mathcal{L}_{3}(\mathbb{Z} ; N)$ with the following property: if a set $\{a, b, c\} \subset \Gamma$ is minimal and consists of linearly independent nodes, then $a, b, c$ is a basis in $\Gamma$. The statement below shows that there are not many lattices without this property.

Lemma 5. For every natural $N$ we have

$$
\#\left(\mathcal{L}_{3}(\mathbb{Z} ; N) \backslash \widetilde{\mathcal{L}}_{3}(\mathbb{Z} ; N)\right) \ll_{\epsilon} N^{1+\epsilon} \quad \forall \epsilon>0 .
$$

Proof. Let $\Gamma \in \mathcal{L}_{3}(\mathbb{Z} ; N) \backslash \widetilde{\mathcal{L}}_{3}(\mathbb{Z} ; N)$. By [16, Theorem 2], there exists a basis of $\Gamma$ which, up to the order of coordinates and up to signs, has the form

$$
\left(a_{1}, a_{2}, a_{3}\right), \quad\left(0, a_{2}, c_{3}\right), \quad\left(0,0,2 c_{3}\right),
$$

where $a_{1}, a_{2}, c_{3}>0, a_{3} \geq 0$. We denote by $L(N)$ the set of lattices in $\mathcal{L}_{3}(\mathbb{Z} ; N)$ generated by vectors of the form (18). By using Lemma 1 it is easy to observe that every lattice in $L(N)$ admits a unique basis of the form (18) that obeys additionally the requirement $a_{3} \in\left[1,2 c_{3}\right]$. Consequently,

$$
\# L(N)=\sum_{2 c_{3} a_{2} \mid N} 2 c_{3} \ll_{\epsilon} N^{1+\epsilon} .
$$

It can easily be checked that

$$
a_{1} b_{2} c_{3} \leq \operatorname{det} A \quad \forall A=\left(\begin{array}{lll}
a_{1} & b_{1} & c_{1} \\
a_{2} & b_{2} & c_{2} \\
a_{3} & b_{3} & c_{3}
\end{array}\right) \in \bigcup_{i=1}^{4} \bar{\Omega}_{i} .
$$

Put

$$
\widetilde{\mathcal{U}}_{+}(N)=\left\{(\gamma, \Gamma) \in \mathcal{U}_{+}(N): \Gamma \in \widetilde{\mathcal{L}}_{3}(\mathbb{Z} ; N)\right\} .
$$

From (2) and (17) it follows that

$$
\#\left(\mathcal{U}_{+}(N) \backslash \tilde{\mathcal{U}}_{+}(N)\right) \ll \ln ^{2} N \cdot \#\left(\mathcal{L}_{3}(\mathbb{Z} ; N) \backslash \widetilde{\mathcal{L}}_{3}(\mathbb{Z} ; N)\right) \ll_{\epsilon} N^{1+\epsilon}
$$


for $N \geq 2$ and every $\epsilon>0$. Thus, it suffices to obtain a formula for the number $\# \tilde{\mathcal{U}}_{+}(N)$. We shall use the following result to estimate the cardinality of subsets of $\tilde{\mathcal{U}}_{+}(N)$.

Lemma 6. Suppose the following conditions are fulfilled:

(a) $U \subset \tilde{\mathcal{U}}_{+}(N), \Omega \subset \bigcup_{i=1}^{4} \bar{\Omega}_{i}, \omega(N)=\Omega \cap \mathrm{GL}_{3}(\mathbb{Z} ; N)$;

(b) there exists an injection $F: \mathbb{Z}^{3 \times 3} \rightarrow \mathbb{Z}^{3 \times 3}$ that preserves the modulus of the determinant of any matrix;

(c) for every pair $(a, \Gamma) \in U$ there exist nodes $b, c \in \Gamma$ such that the set $\{a, b, c\}$ is minimal and $F([a, b, c]) \in \Omega$.

Then

$$
\# U \leq \# \omega(N) .
$$

Proof. We introduce a map $\Phi: U \rightarrow \Omega$ that takes each pair $(a, \Gamma) \in U$ to the matrix $F([a, b, c])$. By (19) and the condition

$$
F([a, b, c]) \in \bigcup_{i=1}^{4} \widetilde{\Omega}_{i},
$$

we obtain $\operatorname{det} F([a, b, c])>0$. Consequently, $\operatorname{det}[a, b, c] \neq 0$, i.e., the nodes $a, b, c$ are linearly independent and, therefore, they constitute a basis of $\Gamma$ (because $\Gamma \in \widetilde{\mathcal{L}}_{3}(\mathbb{Z} ; N)$ ). Then $|\operatorname{det}[a, b, c]|=N$, $\operatorname{det} \Phi(a, \Gamma)=N$, whence $\Phi(a, \Gamma) \in \omega(N)$. It can easily be checked that

$$
\Phi(a, \Gamma)=\Phi\left(a^{\prime}, \Gamma^{\prime}\right) \quad \Longleftrightarrow \quad(a, \Gamma)=\left(a^{\prime}, \Gamma^{\prime}\right) .
$$

Thus, the map $\Phi: U \rightarrow \omega(N)$ is an injection, and (21) is fulfilled.

Let $(a, \Gamma) \in \tilde{\mathcal{U}}_{+}(N)$. We denote

$$
H_{2}(a, \Gamma)=\left\{\gamma \in \mathfrak{M}(\Gamma) \backslash\{ \pm a\}:\left|\gamma_{i}\right| \leq a_{i}, \quad i=1,3\right\} .
$$

We observe immediately that the set $H_{2}(a, \Gamma)$ is nonempty because it contains a node of the form $(0, n, 0)$. Moreover,

$$
\left|\gamma_{2}\right| \geq a_{2} \quad \forall \gamma \in H_{2}(a, \Gamma)
$$

because otherwise $a$ is not minimal.

Also, for every pair $(a, \Gamma) \in \widetilde{\mathcal{U}}_{+}(N)$ we define

$\widetilde{H}_{2}(a, \Gamma)=\left\{\gamma \in \Gamma \backslash\{0, \pm a\}:\left|\gamma_{i}\right| \leq a_{i}, i=1,3\right\} \backslash\left\{\gamma \in \Gamma:\left|\gamma_{i}\right|=a_{i}, i=1,3,\left|\gamma_{2}\right|>a_{2}\right\}$.

Lemma 7. Suppose that $(a, \Gamma) \in \tilde{\mathcal{U}}_{+}(N)$ and a node $b \in H_{2}(a, \Gamma)$ satisfies the inequalities

$$
0<b_{2} \leq\left|\gamma_{2}\right| \quad \forall \gamma \in H_{2}(a, \Gamma) .
$$

Then

$$
b_{2} \leq\left|\gamma_{2}\right| \quad \forall \gamma \in \widetilde{H}_{2}(a, \Gamma),
$$

and the set $\{a, b\}$ is minimal; moreover, either $b_{1} \leq 0$ or $b_{3} \leq 0$.

Proof. We verify (23). Suppose $\gamma \in \Gamma \backslash\{0, \pm a\}$,

$$
\left|\gamma_{1}\right| \leq a_{1},\left|\gamma_{2}\right|<b_{2},\left|\gamma_{3}\right| \leq a_{3}, \gamma \neq \pm a \text {. }
$$

Since $\gamma \neq \pm a$, we have $\gamma \notin \mathfrak{M}(\Gamma)$ (otherwise (22) fails); therefore, there is a node $\eta \in \mathfrak{M}(\Gamma)$ with

$$
\begin{aligned}
\left|\eta_{i}\right| \leq\left|\gamma_{i}\right|, \quad i=1,2,3, \quad \sum_{i=1}^{3}\left|\eta_{i}\right|<\sum_{i=1}^{3}\left|\gamma_{i}\right| \\
\Longrightarrow \quad\left|\eta_{1}\right| \leq a_{1},\left|\eta_{2}\right|<b_{2},\left|\eta_{3}\right| \leq a_{3} .
\end{aligned}
$$


By (22), this is possible only if $\eta= \pm a$. Consequently,

$$
a_{i}=\left|\eta_{i}\right| \leq\left|\gamma_{i}\right| \leq a_{i} \Longrightarrow\left|\gamma_{i}\right|=a_{i}, \quad i=1,3
$$

Thus, $a_{2}<\left|\gamma_{2}\right|$. This proves (23). The minimality of the set $\{a, b\}$ follows from (23). If $b_{1,3}>0$, then the node $\gamma=a-b$ violates the minimality of $\{a, b\}$.

We denote by $V(N)$ the set of $(a, \Gamma) \in \tilde{\mathcal{U}}_{+}(N)$ for which a node $b \in H_{2}(a, \Gamma)$ satisfying (22) is unique. We show that the number of elements not belonging to $V(N)$ is fairly small.

Lemma 8. We have

$$
\# \tilde{\mathcal{U}}_{+}(N)=\# V(N)+O\left(\# \partial \omega_{2}(N)+\# \partial \omega_{4}(N)\right) .
$$

Proof. Suppose that $(a, \Gamma) \in \tilde{\mathcal{U}}_{+}(N) \backslash V(N)$. Then there are $b, b^{\prime} \in H_{2}(a, \Gamma)$ satisfying (22). Clearly, $b_{2}=b_{2}^{\prime}>0$. If $b_{1} b_{1}^{\prime} \geq 0$ and $b_{3} b_{3}^{\prime} \geq 0$, then the node $b-b^{\prime}$ violates the minimality of $a$. Thus, either $b_{1} b_{1}^{\prime}<0$, or $b_{3} b_{3}^{\prime}<0$. Consequently, up to the interchangement of $b$ and $b^{\prime}$, only the following variants are possible:

$$
\begin{aligned}
& b=\left(-b_{1}, b_{2},-b_{3}\right), \\
& b=\left(-b_{1}, b_{2},-b_{3}\right), \\
& b=\left(-b_{1}, b_{2}, b_{3}\right),
\end{aligned}
$$$$
b^{\prime}=\left(-b_{1}^{\prime}, b_{2}, b_{3}^{\prime}\right),
$$$$
b^{\prime}=\left(b_{1}^{\prime}, b_{2},-b_{3}^{\prime}\right) \text {, }
$$$$
b^{\prime}=\left(b_{1}^{\prime}, b_{2},-b_{3}^{\prime}\right),
$$

where $b_{1,3}, b_{1,3}^{\prime} \geq 0$. We introduce the sets $X_{1}, X_{2}$, and $X_{3}$ that consist of $(a, \Gamma) \in$ $\left(\mathcal{U}_{+}(N) \backslash V(N)\right)$ such that $b$ and $b^{\prime}$ have the form (25), (26), or (27) (respectively). Clearly,

$$
\# X_{1}=\# X_{2}
$$

because the interchangement of the 1 st and $3 \mathrm{~d}$ coordinates of nodes generates a bijection between $X_{1}$ and $X_{2}$. It remains to prove that

$$
\# X_{1} \ll \# \partial \omega_{2}(N), \quad \# X_{3} \ll \# \partial \omega_{4}(N) .
$$

Observe at once that the set $\left\{a, b, b^{\prime}\right\}$ is minimal and

$$
\begin{aligned}
b_{1}+b_{1}^{\prime}>a_{1} & \text { for }(a, \Gamma) \in X_{1}, \\
b_{1}+b_{1}^{\prime}>a_{1} \text { or } b_{3}+b_{3}^{\prime}>a_{3} & \text { for }(a, \Gamma) \in X_{3} .
\end{aligned}
$$

(Otherwise the node $b-b^{\prime}$ violates the minimality of $a$.) We introduce the set $X_{31} \subset X_{3}$ $\left(X_{32} \subset X_{3}\right)$ consisting of the pairs $(a, \Gamma)$ for which the nodes $b, b^{\prime}$ satisfy $b_{1}+b_{1}^{\prime}>a_{1}$ $\left(b_{3}+b_{3}^{\prime}>a_{3}\right)$. Then

$$
\# X_{31}=\# X_{32}, \quad \# X_{3} \leq 2 \cdot \# X_{31}
$$

Let $(a, \Gamma) \in X_{1}$. We prove that $b_{3}^{\prime} \geq b_{3}$. Put

$$
\gamma=a-b^{\prime}+b=\left(a_{1}-b_{1}^{\prime}-b_{1}, a_{2}, a_{3}+b_{3}^{\prime}-b_{3}\right) .
$$

Since $a_{1} \leq b_{1}+b_{1}^{\prime} \leq 2 a_{1}$, we have $\left|\gamma_{1}\right| \leq a_{1}$. Since $b_{3} \leq a_{3}$, for $b_{3}^{\prime}<b_{3}$ the node $\gamma$ violates the minimality of $a$. Therefore, $b_{3}^{\prime} \geq b_{3}$. By (29) and the conditions on $b$ and $b^{\prime}$, the matrix $\left[a, b, b^{\prime}-b\right]$ belongs to $\partial \Omega_{2}$. Consequently, the first inequality in (28) holds true (see Lemma 6).

Let $(a, \Gamma) \in X_{31}$. Then $\left[a, b,\left(a-b^{\prime}\right)\right] \in \partial \Omega_{4}$ and, by Lemma 6 , the second inequality in (28) holds true.

On the set $V(N)$, we can introduce a mapping $\Phi_{2}$ that takes any pair $(a, \Gamma) \in V(N)$ to the node $b \in H_{2}(a, \Gamma)$ that satisfies (22). Note that

$$
b_{2}<\left|\gamma_{2}\right| \quad \forall \gamma \in \widetilde{H}_{2}(a, \Gamma), \gamma \neq \pm b,
$$

for $b=\Phi_{2}(a, \Gamma),(a, \Gamma) \in V(N)$. This can be checked much as in the proof of (23). 
We introduce the set

$$
H_{3}(a, \Gamma)=\left\{\gamma \in \mathfrak{M}(\Gamma) \backslash\{ \pm a, \pm b\}:\left|\gamma_{1}\right| \leq a_{1},\left|\gamma_{2}\right| \leq b_{2}\right\} .
$$

It is nonempty (for example, it contains a node of the form $(0,0, n)$ ). Moreover,

$$
\left|\gamma_{3}\right|>a_{3} \quad \forall \gamma \in H_{3}(a, \Gamma),
$$

because otherwise $\gamma$ violates (31).

We shall also employ the set $C(a, \Gamma)$ consisting of the nodes $c$ that satisfy the conditions

$$
c \in H_{3}(a, \Gamma), \quad 0<c_{3} \leq\left|\gamma_{3}\right| \quad \forall \gamma \in H_{3}(a, \Gamma),
$$

and

$$
\begin{aligned}
\Pi_{1}(a) & =\left\{x \in \Gamma:\left|x_{1}\right|=a_{1},\left|a_{i}\right| \leq\left|x_{1}\right|, i=2,3,\left|a_{2}\right|+\left|a_{3}\right|<\left|x_{2}\right|+\left|x_{3}\right|\right\}, \\
\Pi_{2}(b) & =\left\{x \in \Gamma:\left|x_{2}\right|=b_{1},\left|b_{i}\right| \leq\left|x_{i}\right|, i=1,3,\left|b_{1}\right|+\left|b_{3}\right|<\left|x_{1}\right|+\left|x_{3}\right|\right\}, \\
\widetilde{H}_{3}(a, \Gamma) & =\left\{\gamma \in \Gamma \backslash\{0, \pm a, \pm b\}:\left|\gamma_{1}\right| \leq a_{1},\left|\gamma_{2}\right| \leq b_{2}, \quad \gamma \notin \Pi_{1}(a) \cup \Pi_{2}(b)\right\} .
\end{aligned}
$$

Lemma 9. Suppose $(a, \Gamma) \in V(N), b=\Phi_{1}(a, \Gamma), c \in C(a, \Gamma)$. Then

$$
c_{3} \leq\left|\gamma_{3}\right| \quad \forall \gamma \in \widetilde{H}_{3}(a, \Gamma),
$$

the set $\{a, b, c\}$ is minimal, and either $c_{1} \leq 0$ or $c_{2} \leq 0$.

The proof is similar to that of Lemma 7 .

We show that the cases where $b_{1}=0$ or $b_{3}=0$ are fairly rare. Put

$$
V_{0}(N)=\left\{(a, \Gamma): b_{1}=0 \text { or } b_{3}=0\right\},
$$

where $b=\Phi_{1}(a, \Gamma)$.

Lemma 10. We have

$$
\# V_{0}(N) \ll \sum_{i=2}^{4} \# \partial \omega_{i}(N) .
$$

Proof. Since $b_{1} \leq 0$ or $b_{3} \leq 0$ (see Lemma 7), the set $V_{0}(N)$ can be represented in the form

$$
\begin{aligned}
V_{0}(N) & =V_{01} \cup V_{01}^{\prime} \cup V_{02} \cup V_{02}^{\prime}, \\
V_{01} & =\left\{(a, \Gamma) \in V(N): b=\left(0, b_{2}, b_{3}\right), b_{3} \geq 0\right\}, \\
V_{01}^{\prime} & =\left\{(a, \Gamma) \in V(N): b=\left(b_{1}, b_{2}, 0\right), b_{1} \geq 0\right\}, \\
V_{02} & =\left\{(a, \Gamma) \in V(N): b=\left(-b_{1}, b_{2}, 0\right), b_{1}>0\right\}, \\
V_{02}^{\prime} & =\left\{(a, \Gamma) \in V(N): b=\left(0, b_{2},-b_{3}\right), b_{3}>0\right\},
\end{aligned}
$$

where $b=\Phi_{1}(a, \Gamma)$. We have $b_{2}>0$ in all cases. Observe that

$$
\# V_{01}=\# V_{01}^{\prime}, \quad \# V_{02}=\# V_{02}^{\prime}
$$

(the transformation interchanging the first and the third coordinates of nodes is a bijection between $V_{01}$ and $V_{01}^{\prime}$, and also between $V_{02}$ and $V_{02}^{\prime}$ ). Thus, it suffices to estimate the cardinalities of $V_{01}$ and $V_{02}$.

We estimate $\# V_{01}$. Let $(a, \Gamma) \in V_{01}$. Then $b=\left(0, b_{2}, b_{3}\right), a_{3} \geq b_{3} \geq 0, b_{2} \geq a_{2}>0$. The node $\gamma=a-b$ satisfies the relations

$$
\left|\gamma_{1}\right|=a_{1}, \quad\left|\gamma_{2}\right|=b_{2}-a_{2}<b_{2}, \quad\left|\gamma_{3}\right|=a_{3}-b_{3} \leq a_{3}
$$

By (31), it follows that either $\gamma \in \Pi_{1}(a)$ or $\gamma= \pm a$. Therefore,

$$
\gamma_{2} \geq a_{2},\left|\gamma_{3}\right| \geq a_{3} \Longrightarrow b_{2} \geq 2 a_{2}, b_{3}=0 \text {. }
$$


Thus, $b=\left(0, b_{2}, 0\right), b_{2} \geq 2 a_{2}$. We choose $c \in C(a, \Gamma)$ arbitrarily. Then either $c_{1} \leq 0$ or $c_{2} \leq 0$ (see Lemma 9). We show that the case where $c=\left(c_{1},-c_{2}, c_{3}\right), c_{1,3}>0, c_{2} \geq 0$ is impossible. Indeed, in this case the node $\gamma=a-c-b$ satisfies the relations

$$
\left|\gamma_{1}\right|=a_{1}-c_{1}<a_{1}, \quad\left|\gamma_{2}\right|=\left|a_{2}+c_{2}-b_{2}\right|<b_{2}, \quad\left|\gamma_{3}\right|=c_{3}-a_{3}<c_{3}
$$

(the second inequality follows from the estimates $a_{2}>0 ; 2 a_{2}, c_{2} \leq b_{2}$ ). By the minimality of $\{a, b, c\}$, this is possible only if $\gamma=0$. However, in this case the node $-c=b-a$ contradicts the choice of $b$. Thus, only the following two cases may occur:

1) $c=\left(-c_{1}, c_{2}, c_{3}\right), c_{1,2,3} \geq 0$, then $[a, b, c] \in \partial \Omega_{2}$;

2) $c=\left(-c_{1},-c_{2}, c_{3}\right), c_{1,2,3} \geq 0$, then $[a, b, c] \in \partial \Omega_{3}$.

So, we have proved that

$$
\# V_{01}=\# V_{01}^{\prime} \leq \# \partial \omega_{2}(N)+\# \partial \omega_{3}(N)
$$

(see Lemma 6).

We estimate $\# V_{02}$. Let $(a, \Gamma) \in V_{02}$. Then $b=\left(-b_{1}, b_{2}, 0\right), b_{1,2}>0$. Take an arbitrary node $c \in C(a, b)$. The following cases are possible.

If $c=\left(-c_{1},-c_{2}, c_{3}\right), c_{1,2} \geq 0$, then $[a, b, c] \in \partial \Omega_{3}$.

Let $c=\left(-c_{1}, c_{2}, c_{3}\right), c_{1,2} \geq 0$. Then the node $\gamma=a+b-c$ satisfies the relations

$$
\left|\gamma_{1}\right|=a_{1}-b_{1}+c_{1},\left|\gamma_{2}\right|=b_{2}+a_{2}-c_{2},\left|\gamma_{3}\right|=c_{3}-a_{3}<c_{3} .
$$

Therefore, either $c_{1} \geq b_{1}$ or $a_{2} \geq c_{2}$ (otherwise $0<\left|\gamma_{1}\right|<a_{1},\left|\gamma_{2}\right|<b_{2}$, and the minimality of $\{a, b, c\}$ is violated). Thus, $[a, b, c] \in \partial \Omega_{2}$.

Let $c=\left(c_{1},-c_{2}, c_{3}\right), c_{1}>0, c_{2} \geq 0$. Then the node $\gamma=a-c$ satisfies the relations

$$
\left|\gamma_{1}\right|=a_{1}-c_{1}<a_{1},\left|\gamma_{2}\right|=a_{2}+c_{2},\left|\gamma_{3}\right|=c_{3}-a_{3}<c_{3}
$$

and $a_{2}+c_{2}=b_{2}$ by the minimality of $\{a, b, c\}$. We put $\eta=a-b-c$. Then

$$
\left|\eta_{1}\right|=a_{1}+b_{1}-c_{1}>0,\left|\eta_{2}\right|=a_{2}+c_{2}-b_{2}=0,\left|\eta_{3}\right|=c_{3}-a_{3}<c_{3} .
$$

Thus, $b_{1} \geq c_{1}$ (otherwise $\{a, b, c\}$ ) is not minimal), $[a, b, c] \in \partial \Omega_{4}$.

We have proved that

$$
\# V_{02}=\# V_{02}^{\prime} \ll \sum_{i=2}^{4} \# \partial \omega_{i}(N) .
$$

We split the set $V(N) \backslash V_{0}(N)$ into mutually disjoint parts:

$$
\begin{aligned}
& V_{1}(N)=\left\{(a, \Gamma) \in V(N): \Phi_{1}(a, \Gamma)=\left(-b_{1}, b_{2},-b_{3}\right)\right\}, \\
& V_{2}(N)=\left\{(a, \Gamma) \in V(N): \Phi_{1}(a, \Gamma)=\left(-b_{1}, b_{2}, b_{3}\right)\right\}, \\
& V_{3}(N)=\left\{(a, \Gamma) \in V(N): \Phi_{1}(a, \Gamma)=\left(b_{1}, b_{2}-b_{3}\right)\right\},
\end{aligned}
$$

where $b_{1,2,3}>0$. Then

$$
V(N)=\bigcup_{i=0}^{3} V_{i}(N)
$$

Since $\# V_{2}(N)=\# V_{3}(N)$ (the map interchanging the first and the third coordinates of nodes is a bijection between $V_{2}(N)$ and $V_{3}(N)$ ), from (24) and (34) it follows that

$$
\# \tilde{\mathcal{U}}_{+}(N)=\# V_{1}(N)+2 \# V_{2}(N)+O\left(\sum_{i=2}^{4} \# \partial \omega_{i}(N)\right) \text {. }
$$

We put

$$
\begin{aligned}
& \tilde{V}_{1}(N)=\left\{(a, \Gamma) \in V_{1}(N): c_{1}<0 \forall c \in C(a, \Gamma)\right\}, \\
& \tilde{V}_{2}(N)=\left\{(a, \Gamma) \in V_{2}(N): c_{2}<0 \forall c \in C(a, \Gamma)\right\}
\end{aligned}
$$


and prove that the collection of elements not belonging to these sets has a "relatively small" cardinality.

Lemma 11. We have

$$
\begin{aligned}
& \# V_{1}(N)=\# \widetilde{V}_{1}(N)+O\left(\# \partial \omega_{1}(N)+\# \partial \omega_{2}(N)\right), \\
& \# V_{2}(N)=\# \widetilde{V}_{1}(N)+O\left(\# \partial \omega_{3}(N)\right)
\end{aligned}
$$

Proof. Suppose that $(a, \Gamma) \in V_{1}(N) \backslash \widetilde{V}_{1}(N)$, and let $b=\Phi_{1}(a, \Gamma)$. Then

$$
b=\left(-b_{1}, b_{2},-b_{3}\right), \quad b_{1,2,3}>0
$$

and there exists a node $c \in C(a, \Gamma)$ with $c_{1} \geq 0$; by Lemma 9 , either $c_{1}=0$ or $c_{2} \leq 0$. Consequently, the following 5 cases are possible.

1. If $c=\left(0,0, c_{3}\right)$, then $[a, b, c] \in \partial \Omega_{2}$.

2. If $c=\left(0,-c_{2}, c_{3}\right)$ and $c_{2}>0$, then the node $\gamma=b+c$ satisfies the relations

$$
\left|\gamma_{1}\right|=b_{1} \leq a_{1}, \quad\left|\gamma_{2}\right|=b_{2}-c_{2}<b_{2}, \quad\left|\gamma_{3}\right|=c_{3}-b_{3}<c_{3} ;
$$

therefore, $a_{1}=b_{1}$ (otherwise the minimality of $\{a, b, c\}$ is violated) and $[a, b, c] \in \partial \Omega_{1}$.

3. Let $c=\left(0, c_{2}, c_{3}\right)$ with $c_{2,3}>0$, then the node $\gamma=a-c$ satisfies the relations

$$
\left|\gamma_{1}\right|=a_{1}, \quad\left|\gamma_{2}\right|=\left|a_{2}-c_{2}\right|<b_{2}, \quad\left|\gamma_{3}\right|=c_{3}-a_{3}<c_{3},
$$

and formula (33) implies that

$$
a_{2} \leq\left|\gamma_{2}\right|, a_{3} \leq\left|\gamma_{3}\right| \quad \Longrightarrow \quad c_{2} \geq 2 a_{2}, c_{3}>2 a_{3} .
$$

Put $\eta=a+b-c$. Then

$$
\left|\eta_{1}\right|=a_{1}-b_{1}<a_{1}, \quad\left|\eta_{2}\right|=a_{2}+b_{2}-c_{2}<b_{2}, \quad\left|\eta_{3}\right|=c_{3}+b_{3}-a_{3} \leq c_{3} .
$$

Therefore, $b_{3}=a_{3}$ and the matrix $[a, b, c-a]$ belongs to $\partial \Omega_{2}$.

4. Let $c=\left(c_{1}, 0, c_{3}\right)$ with $c_{1}>0$. The node $\gamma=a-c$ satisfies the relations

$$
\left|\gamma_{1}\right|=a_{1}-c_{1}<a_{1}, \quad\left|\gamma_{2}\right|=a_{2}, \quad\left|\gamma_{3}\right|=c_{3}-a_{3}<c_{3} .
$$

By (33), this is possible only if $\gamma \in \Pi_{2}(b)$, i.e.,

$$
b_{i} \leq\left|\gamma_{i}\right|, i=1,3,\left|\gamma_{2}\right|=b_{2} \quad \Longrightarrow \quad a_{2}=b_{2}, \quad a_{3}+b_{3} \leq c_{3} .
$$

Next, consider the node $\theta=a-b-c$. It satisfies the relations

$$
0<\left|\theta_{1}\right|=a_{1}+b_{1}-c_{1},\left|\theta_{2}\right|=0,\left|\theta_{3}\right|=c_{3}-\left(a_{3}+b_{3}\right)<c_{3} .
$$

Consequently, $b_{1}>c_{1}$ (otherwise (33) fails). Then the node $\eta=b+c$ contradicts (33). We see that this case is impossible.

5 . The case where $c=\left(c_{1},-c_{2}, c_{3}\right), c_{1,2}>0$, is also impossible, because then the node $b+c$ violates the minimality of $\{a, b, c\}$.

Applying Lemma 6, we arrive at

$$
\#\left(V_{1}(N) \backslash \tilde{V}_{1}(N)\right) \ll \# \partial \omega_{1}(N)+\# \partial \omega_{2}(N) .
$$

This proves (36).

Now, we prove (37). Suppose $(a, \Gamma) \in V_{2}(N) \backslash \widetilde{V}_{2}(N)$, and let $b=\Phi_{1}(a, \Gamma), b=$ $\left(-b_{1}, b_{2}, b_{3}\right)$ with $b_{1,2,3}>0$. Then there exists $c \in C(a, \Gamma)$ with the property that $c_{2} \geq 0$, and moreover, either $c_{2}=0$ or $c_{1} \leq 0$ (by Lemma 9). The following 4 cases are possible.

1. If $c=\left(-c_{1}, 0, c_{3}\right), c_{1} \geq 0$, then $[a, b, c] \in \partial \Omega_{3}$.

2. If $c=\left(-c_{1}, c_{2}, c_{3}\right), c_{1,2}>0$, then the node $b-c$ violates the minimality of $\{a, b, c\}$.

3. Let $c=\left(c_{1}, 0, c_{3}\right)$ with $c_{1}>0$. Then the node $\gamma=a-c$ satisfies the relations

$$
\left|\gamma_{1}\right|=a_{1}-c_{1}<a_{1}, \quad\left|\gamma_{2}\right|=a_{2}, \quad\left|\gamma_{3}\right|=c_{3}-a_{3}<c_{3} .
$$

Thus, $c_{3} \geq 2 a_{3}$ (otherwise $\left.a \notin \mathfrak{M}(\Gamma)\right)$ and $[a, b, c-b] \in \partial \Omega_{3}$. 
4. It only remains to consider the case where $c=\left(0, c_{2}, c_{3}\right), c_{2}>0$. The nodes $\gamma=a-c$ and $\eta=b-c$ satisfy the relations

$$
\begin{array}{ll}
\left|\gamma_{1}\right|=a_{1}, & \left|\gamma_{2}\right|=\left|a_{2}-c_{2}\right|<b_{2}, \quad\left|\gamma_{3}\right|=c_{3}-a_{3}<c_{3}, \\
\left|\eta_{1}\right|=\left|b_{1}\right|, \quad\left|\eta_{2}\right|=b_{2}-c_{2}<b_{2}, & \left|\eta_{3}\right|=c_{3}-b_{3}<c_{3} .
\end{array}
$$

By (33), this is possible only if $\eta, \gamma \in \Pi_{1}(a)$,

$$
\begin{aligned}
a_{i} \leq\left|\gamma_{i}\right|,\left|\eta_{i}\right|, \quad i=2,3, \quad\left|\eta_{1}\right| & =\left|\gamma_{1}\right|=a_{1} \\
& \Longrightarrow a_{2} \geq 2 c_{2}, \quad c_{3} \geq 2 a_{3}, \quad a_{1}=b_{1}, \quad b_{2} \geq a_{2}+c_{2}, \quad c_{3} \geq a_{3}+b_{3},
\end{aligned}
$$

and the matrix $[a, b, c-a]$ belongs to $\partial \Omega_{3}$.

Applying Lemma 6, we obtain

$$
\#\left(V_{2}(N) \backslash \tilde{V}_{2}(N)\right) \ll \# \partial \omega_{3}(N) .
$$

This proves formula (37).

Let $(a, \Gamma) \in \tilde{V}_{1}(N) \cup \tilde{V}_{2}(N)$. If there exist nodes $c, c^{\prime} \in C(a, \Gamma)$ with $c_{i} c_{i}^{\prime} \geq 0, i=1,2$, then $\gamma=c-c^{\prime}$ satisfies the relations

$$
\left|\gamma_{1}\right| \leq a_{1}, \quad\left|\gamma_{2}\right| \leq b_{2}, \quad \gamma_{3}=0 .
$$

This contradicts (31). Therefore, either $C(a, \Gamma)$ is a singleton or it consists of two elements one of which is a node of the form $c=\left(-c_{1},-c_{2}, c_{3}\right), c_{1,2}>0$. Consequently, we can introduce a mapping $\Phi_{2}: \tilde{V}_{1}(N) \cup \tilde{V}_{2}(N) \rightarrow \mathbb{Z}^{3}$ acting in accordance with the rule $\Phi_{2}(a, \Gamma)=c$, where $c \in C(a, \Gamma)$ is as above. If there are two elements with these property, we choose $c$ to be of the form $c=\left(-c_{1}, c_{2},-c_{3}\right)$ with $c_{1,2,3}>0$.

Lemma 12. We have

$$
\# \tilde{V}_{1}(N) \leq \# \bar{\omega}_{1}(N)+\# \bar{\omega}_{2}(N), \quad \# \tilde{V}_{2}(N) \leq \# \bar{\omega}_{3}(N)+\# \bar{\omega}_{4}(N) .
$$

Proof. Suppose that $(a, \Gamma) \in \widetilde{V}_{1}(N)$, let $b=\Phi_{1}(a, \Gamma)$, and let $c=\Phi_{2}(a, \Gamma)$. Then $b=\left(-b_{1}, b_{2},-b_{3}\right)$ with $b_{1,2,3}>0$. Two cases are possible.

1. Let $c=\left(-c_{1},-c_{2}, c_{3}\right), c_{1,2,3}>0$. Put $\gamma=b+c$. Then

$$
\left|\gamma_{1}\right|=\left|b_{1}+c_{1}\right|, \quad\left|\gamma_{2}\right|=\left|b_{2}-c_{2}\right|<b_{2}, \quad\left|\gamma_{3}\right|=c_{3}-b_{3}<c_{3} .
$$

Consequently, $a_{1} \leq b_{1}+c_{1}$ (otherwise $\gamma$ violates the minimality of $\{a, b, c\}$ ) and $[a, b, c] \in$ $\bar{\Omega}_{1}$.

2. Suppose $c=\left(-c_{1}, c_{2}, c_{3}\right), c_{1,3}>0, c_{2} \geq 0$. In this case $C(a, \Gamma)$ is a singleton, and, as in Lemma 7, we prove that

$$
c_{3}<\left|\gamma_{3}\right| \forall \gamma \in \widetilde{H}_{3}(a, \Gamma), \gamma \neq \pm c
$$

Put $\gamma=-b-a+c$. Then

$$
\left|\gamma_{1}\right|=\left|a_{1}+\left(c_{1}-b_{1}\right)\right|, \quad\left|\gamma_{2}\right|=\left|b_{2}+\left(a_{2}-c_{2}\right)\right|, \quad 0<\left|\gamma_{3}\right|=c_{3}+\left(b_{3}-a_{3}\right) \leq c_{3} .
$$

Consequently, either $c_{1} \geq b_{1}$ or $a_{2} \geq c_{2}$ (otherwise $\gamma$ violates (39)) and $[a, b, c] \in \bar{\Omega}_{2}$. By Lemma 6, the first estimate in (38) is true.

Let $(a, \Gamma) \in \widetilde{V}_{2}(N)$, let $b=\Phi_{1}(a, \Gamma)$, and let $c=\Phi_{2}(a, \Gamma)$. Then $b=\left(-b_{1}, b_{2}, b_{3}\right)$, $b_{1,2,3}>0$. Two cases are possible.

1. Suppose $c=\left(-c_{1},-c_{2}, c_{3}\right), c_{1,2,3}>0$. Then $[a, b, c] \in \bar{\Omega}_{3}$.

2. Suppose $c=\left(-c_{1}, c_{2}, c_{3}\right), c_{1,3}>0, c_{2} \geq 0$. Then the set $C(a, \Gamma)$ is a singleton and (39) is fulfilled. Put $\gamma=-a+c$, then

$$
\left|\gamma_{1}\right|=\left|a_{1}-c_{1}\right|<a_{1}, \quad\left|\gamma_{2}\right|=\left|a_{2}+c_{2}\right|, \quad\left|\gamma_{3}\right|=c_{3}-a_{3}<c_{3}
$$


Therefore, $a_{2}+c_{2} \geq b_{2}$ (otherwise $\gamma$ violates the minimality of $\{a, b, c\}$ ). We prove that $b_{1} \geq c_{1}$. For this, consider the node $\eta=-a+b+c$. We have

$$
\begin{aligned}
& \left.\left|\eta_{1}\right|=\left|a_{1}+\left(b_{1}-c_{1}\right)\right|, \quad\left|\eta_{2}\right|=a_{2}+c_{2}-b_{2} \leq b_{2} \quad \text { (because } a_{2}+c_{2} \leq 2 b_{2}\right), \\
& \left.\left|\eta_{3}\right|=c_{3}+\left(b_{3}-a_{3}\right) \leq c_{3} \quad \text { (because } b_{3} \leq a_{3}\right) .
\end{aligned}
$$

If $a_{2}+c_{2}<2 b_{2}$, then $\left|\eta_{2}\right|<b_{2}$, and, therefore, $b_{1} \geq c_{1}$ (otherwise $\eta$ violates condition (39) ). Let $a_{2}+c_{2}=2 b_{2}$. Then $a_{2}=b_{2}=c_{2}$, consequently, $\left|a_{1}\right|=\left|b_{1}\right|,\left|a_{3}\right|=\left|b_{3}\right|$ (otherwise $b$ violates the minimality of $a$ ), and $b_{1}=a_{1} \geq c_{1}$. Hence, $[a, b, c] \in \bar{\Omega}_{4}$. Applying Lemma 6, we obtain the second inequality in (38).

Now, we prove the existence of injections

$$
\omega_{1}(N) \cup \omega_{2}(N) \rightarrow \tilde{V}_{1}(N), \quad \omega_{3}(N) \cup \omega_{4}(N) \rightarrow \tilde{V}_{2}(N) .
$$

Lemma 13. Let a lattice $\Gamma$ be generated by vectors $a, b, c$. Consider the matrix $A=$ $[a, b, c]$. Then:

if $A \in \omega_{1}(N) \cup \omega_{2}(N)$, then $(a, \Gamma) \in \widetilde{V}_{1}(N)$, and

if $A \in \omega_{3}(N) \cup \omega_{4}(N)$, then $(a, \Gamma) \in \widetilde{V}_{2}(N)$;

furthermore, $b=\Phi_{1}(a, \Gamma)$ and $c=\Phi_{2}(a, \Gamma)$ in both cases.

Proof. It suffices to show that there are no integers $m, n$, and $k$ with the property that the node

$$
\gamma=m a+n b+k c
$$

satisfies the conditions

$$
\left|\gamma_{1}\right| \leq a_{1}, \quad\left|\gamma_{2}\right| \leq b_{2}, \quad\left|\gamma_{3}\right| \leq c_{3}, \quad \gamma \notin\{0, \pm a, \pm b, \pm c\} .
$$

The numbers $m, n$, and $k$ will be called coefficients.

If two coefficients are equal to zero and the third is of modulus greater than 1 , then either $\left|\gamma_{1}\right|>b_{1}$, or $\left|\gamma_{2}\right|>b_{2}$, or $\left|\gamma_{3}\right|>c_{3}$.

Suppose that precisely one of the coefficients is equal to zero. Then:

$$
\begin{aligned}
& \text { if } m \cdot n>0, k=0 \text {, then }\left|\gamma_{2}\right|=\left|m a_{2}\right|+\left|n b_{2}\right|>\left|b_{2}\right| \text {; } \\
& \text { if } m \cdot n<0, k=0 \text {, then }\left|\gamma_{1}\right|=\left|m a_{1}\right|+\left|n b_{1}\right|>\left|a_{1}\right| \text {; } \\
& \text { if } m \cdot k>0, n=0 \text {, then }\left|\gamma_{3}\right|=\left|m a_{3}\right|+\left|k c_{3}\right|>\left|c_{3}\right| \text {; } \\
& \text { if } m \cdot k<0, n=0 \text {, then } \\
& \left|\gamma_{1}\right|=\left|m a_{1}\right|+\left|k c_{1}\right|>\left|a_{1}\right| \quad \text { for } A \in \omega_{1}(N) \cup \omega_{2}(N) \cup \omega_{3}(N) \text {, } \\
& \left|\gamma_{2}\right|=\left|m a_{2}\right|+\left|k c_{2}\right| \geq\left|a_{2}\right|+\left|c_{2}\right|>\left|b_{2}\right| \quad \text { for } A \in \omega_{4}(N) \text {; } \\
& \text { if } m=0, n \cdot k>0 \text {, then }
\end{aligned}
$$$$
\begin{array}{lll}
\left|\gamma_{1}\right|=\left|n b_{1}\right|+\left|k c_{1}\right| \geq\left|b_{1}\right|+\left|c_{1}\right|>a_{1} & \text { for } & A \in \omega_{1}(N), \\
\left|\gamma_{2}\right|=\left|n b_{2}\right|+\left|k c_{2}\right|>b_{2} & \text { for } \quad A \in \omega_{2}(N), \\
\left|\gamma_{3}\right|=\left|n b_{3}\right|+\left|k c_{3}\right|>c_{3} & \text { for } \quad A \in \omega_{3}(N) \cup \omega_{4}(N),
\end{array}
$$

if $m=0, n \cdot k<0$, then

$$
\begin{aligned}
& \left|\gamma_{3}\right|=\left|n b_{3}\right|+\left|k c_{3}\right|>c_{3} \quad \text { for } A \in \omega_{1}(N) \cup \omega_{2}(N), \\
& \left|\gamma_{2}\right|=\left|n b_{2}\right|+\left|k c_{2}\right|>b_{2} \quad \text { for } A \in \omega_{3}(N) \cup \omega_{4}(N) .
\end{aligned}
$$

It remains to consider the cases where $m, n, k \neq 0$. There is no loss of generality in assuming that $m>0$. Four cases are possible.

1. Let $n, k>0$.

If $A \in \omega_{1}(N)$, then

$$
\begin{aligned}
& \left|\gamma_{3}\right|=\left|m a_{3}\right|-\left|n b_{3}\right|+\left|k c_{3}\right| \geq|| k c_{3}|-|(n-1) b_{3} \mid>c_{3} \\
& \left|\gamma_{2}\right|=\left|m a_{2}\right|+\left|n b_{2}\right|-\left|k c_{2}\right|>b_{2}
\end{aligned}
$$


If $A \in \omega_{2}(N)$, then $\left|\gamma_{2}\right|=\left|m a_{2}\right|+\left|n b_{2}\right|+\left|k c_{2}\right|>\left|b_{2}\right|$.

If $A \in \omega_{3}(N) \cup \omega_{4}(N)$, then $\left|\gamma_{3}\right|=\left|m a_{3}\right|+\left|n b_{3}\right|+\left|k c_{3}\right|>\left|c_{3}\right|$.

2. Let $n, k<0$.

If $A \in \omega_{1}(N) \cup \omega_{2}(N) \cup \omega_{3}(N)$, then $\left|\gamma_{1}\right|=\left|m a_{1}\right|+\left|n b_{1}\right|+\left|k c_{1}\right|>\left|a_{1}\right|$.

If $A \in \omega_{4}(N)$, then

$$
\begin{array}{ll}
\left|\gamma_{1}\right|=\left|m a_{1}\right|+\left|n b_{1}\right|-\left|k c_{1}\right|>a_{1} & \text { for } m>k \text { or } n \geq k, \\
\left|\gamma_{3}\right|=-\left|m a_{3}\right|+\left|n b_{3}\right|+\left|k c_{3}\right|>c_{3} & \text { for } m<k, \\
\left|\gamma_{2}\right|=\left|m a_{2}\right|-\left|n b_{2}\right|+\left|m c_{2}\right|>b_{2} & \text { for } k=m>n .
\end{array}
$$

3. Let $n<0$ and $k>0$.

If $A \in \omega_{1}(N) \cup \omega_{2}(N)$, then $\left|\gamma_{3}\right|=\left|m a_{3}\right|+\left|n b_{3}\right|+\left|k c_{3}\right|>\left|c_{3}\right|$.

If $A \in \omega_{3}(N)$, then

$$
\begin{array}{ll}
\left|\gamma_{2}\right|=-\left|m a_{2}\right|+\left|n b_{2}\right|+\left|k c_{2}\right|>b_{2} & \text { for } n>m, \\
\left|\gamma_{3}\right|=\left|m a_{3}\right|-\left|n b_{3}\right|+\left|k c_{3}\right|>c_{3} & \text { for } n \leq m .
\end{array}
$$

If $A \in \omega_{4}(N)$, then $\left|\gamma_{1}\right|=\left|m a_{1}\right|+\left|n b_{1}\right|+\left|k c_{1}\right|>\left|a_{1}\right|$.

4. Let $n>0$ and $k<0$.

If $A \in \omega_{1}(N) \cup \omega_{3}(N) \cup \omega_{4}(N)$, then $\left|\gamma_{2}\right|=\left|m a_{2}\right|+\left|n b_{2}\right|+\left|k c_{2}\right|>\left|b_{2}\right|$.

If $A \in \omega_{2}(N)$, then

$$
\begin{array}{ll}
\left|\gamma_{1}\right|=\left|m a_{1}\right|-\left|n b_{1}\right|+\left|k c_{1}\right|>a_{1} & \text { for } m>n, \\
\left|\gamma_{2}\right|=\left|m a_{2}\right|+\left|n b_{2}\right|-\left|k c_{2}\right|>b_{2} & \text { for } n>k, \\
\left|\gamma_{3}\right|=-\left|m a_{3}\right|+\left|n b_{3}\right|+\left|k c_{3}\right|>c_{3} & \text { for } k>m, \\
\left|\gamma_{1}\right|>a_{1} \text { or }\left|\gamma_{2}\right|>b_{2} & \text { for } k=m=n .
\end{array}
$$

Proof of Lemma 4. By (20), (35), (36), and (37), it suffices to prove that

$$
\begin{aligned}
& \# \tilde{V}_{1}(N)=\# \omega_{1}(N)+\# \omega_{2}(N)+O\left(\# \partial \omega_{1}(N)+\# \partial \omega_{2}(N)\right), \\
& \# \widetilde{V}_{2}(N)=\# \omega_{3}(N)+\# \omega_{4}(N)+O\left(\# \partial \omega_{3}(N)+\# \partial \omega_{4}(N)\right) .
\end{aligned}
$$

From (38) it follows that

$$
\begin{aligned}
& \# \tilde{V}_{1}(N) \leq \# \omega_{1}(N)+\# \omega_{2}(N)+O\left(\# \partial \omega_{1}(N)+\# \partial \omega_{2}(N)\right), \\
& \# \tilde{V}_{2}(N) \leq \# \omega_{3}(N)+\# \omega_{4}(N)+O\left(\# \partial \omega_{3}(N)+\# \partial \omega_{4}(N)\right) .
\end{aligned}
$$

By Lemma 13, the injections (40) exist (they act by the formula $A \rightarrow(a, \Gamma)$, where $a$ is the first column of the matrix $A$ and the lattice $\Gamma$ is generated by the columns of $A$ ). Taking into account the relations

$$
\omega_{1}(N) \cap \omega_{2}(N)=\varnothing, \quad \omega_{3}(N) \cap \omega_{4}(N)=\varnothing,
$$

we obtain

$$
\# \omega_{1}(N)+\# \omega_{2}(N) \leq \# \tilde{V}_{1}(N), \quad \# \omega_{3}(N)+\# \omega_{4}(N) \leq \# \tilde{V}_{2}(N) .
$$

Now, (42) is a consequence of (43) and (41).

\section{$\S 4$. The number of integral matrices in a Given domain}

By Lemma 4, our initial problem reduces to the calculation of the number of integral matrices that lie in a certain domain $\Omega_{i}$ (not depending on $N$ ) with determinant belonging to $[1, N]$. It can easily be observed that this is equivalent to the problem about the number of integral points in a fixed domain. In the two-dimensional case, the following formula is well known for an arbitrary domain $U$ with boundary $\partial U$ :

$$
\#\left(U \cap \mathbb{Z}^{2}\right)=\text { meas } U+O(\text { meas } \partial U+1)
$$


where meas is Lebesgue measure. For a convex domain, this is a consequence of the Jarnik inequality. The proof for nonconvex domains can be found, e.g., in [18]. It is easily seen that the formula fails for $s$-dimensional domains with $s \geq 3$. For that, it suffices to consider the cylinder

$$
\left\{(x, y, z) \in \mathbb{R}^{3}: x^{2}+y^{2}<L^{-4}, 0<z<L\right\}
$$

as $L \rightarrow+\infty$. In the present paper, the following result will suffice. Put

$$
\begin{aligned}
\|x\|_{\infty} & =\max _{1 \leq i \leq s}\left|x_{i}\right| \quad \forall x \in \mathbb{R}^{s}, \\
\rho_{\infty}(X, Y) & =\inf _{x \in X, y \in Y}\|x-y\|_{\infty} \quad \forall X, Y \subset \mathbb{R}^{s}, \\
B(S, r) & =\left\{x \in \mathbb{R}^{s}: \rho_{\infty}(x, S)<r\right\} \quad \forall S \subset \mathbb{R}^{s}, r>0 .
\end{aligned}
$$

Lemma 14. Let $U$ be a finitely connected Lebesgue measurable subset of $\mathbb{R}^{s}(s \geq 2)$. Then

$$
\left|\#\left(U \cap \mathbb{Z}^{s}\right)-\operatorname{meas} U\right| \leq 2^{s} \cdot \text { meas } B(\partial U, 1) .
$$

Proof. Put

$$
\begin{aligned}
\Pi(x) & =\left\{y \in \mathbb{R}^{s}: y_{i}=x_{i}+t_{i}, \quad t_{i} \in[0,1), i=1, \ldots, s\right\} \quad \forall x \in \mathbb{Z}^{s}, \\
X & =\left\{x \in \mathbb{Z}^{s}: \Pi(x) \subset U\right\}, \quad X^{\prime}=\left(U \cap \mathbb{Z}^{s}\right) \backslash X .
\end{aligned}
$$

Clearly,

$$
0 \leq \text { meas } U-\# X \leq \operatorname{meas}\left\{x \in U: \rho_{\infty}(x, \partial U)<1\right\} \leq \text { meas } B(\partial U, 1) .
$$

Since

$$
\# X^{\prime} \leq 2^{s} \cdot \#\left\{x \in \mathbb{Z}^{s}: \Pi(x) \cap \partial U \neq \varnothing\right\} \leq 2^{s} \text { meas } B(\partial U, 1)
$$

we have

$$
\left|\operatorname{meas} U-\#\left(U \cap \mathbb{Z}^{s}\right)\right|=\left|(\operatorname{meas} U-\# X)-\# X^{\prime}\right| \leq 2^{s} \text { meas } B(\partial U, 1) .
$$

To an arbitrary matrix $A \in \mathrm{GL}_{3}(\mathbb{R})$ with entries $a_{i j}$, we assign the point

$$
A=\left(a_{11}, a_{12}, a_{13}, a_{21}, a_{22}, a_{23}, a_{31}, a_{32}, a_{33}\right) \in \mathbb{R}^{9} .
$$

For $\Omega \subset \mathrm{GL}_{3}(\mathbb{R})$, meas $\Omega$ denotes the Lebesgue measure of $\Omega$,

$$
\begin{aligned}
& \Omega([1 ; N])=\left\{A \in \Omega: 1 \leq a_{i i}, \quad i=1,2,3, \quad \operatorname{det} A \in[1, N]\right\}, \\
& \omega([1 ; N])=\Omega([1 ; N]) \cap \mathrm{GL}_{3}(\mathbb{Z}) .
\end{aligned}
$$

A surface $L \subset \mathbb{R}^{s}$ is said to belong to the class $\widetilde{C}^{1}$ if $L \subset \widetilde{L}$, where $\widetilde{L}$ is the graph of a function $f \in C^{1}\left(\mathbb{R}^{s-1}\right)$ whose derivatives are uniformly bounded on $\mathbb{R}^{s-1}$.

A surface $L \subset \mathbb{R}^{s}$ is said to be piecewise smooth if it is composed of a fixed number of surfaces belonging to $\widetilde{C}^{1}$.

Lemma 15. Let $\Omega$ be a connected set in $\mathrm{GL}_{3}(\mathbb{R})$ (independent of $N$ ) with piecewise smooth boundary, and suppose that there exists a constant $C=C(\Omega)$ such that

$$
\begin{aligned}
\left|a_{i j}\right|<C \cdot a_{i i}, \quad i, j=1,2,3, \\
\operatorname{det} A \geq C \cdot a_{11} a_{22} a_{33} \quad \forall A=\left(\left(a_{i j}\right)\right) \in \Omega .
\end{aligned}
$$

Then

$$
\# \omega([1 ; N])=\operatorname{meas} \Omega([1 ; N])+O_{\Omega}\left(N^{3} \ln N\right)
$$

for $N>1$. 
Proof. We denote by $D_{i j}=D_{i j}(A)$ the determinant obtained after elimination the $i$ th row and $j$ th column from a square matrix $A ; D(A)=\operatorname{det} A$.

By using (45), it can easily be checked that $\Omega$ can be represented as the union of 3 mutually nonintersecting sets $\Omega^{(i)}(i=1,2,3)$ with piecewise smooth boundary, in such a way that

$$
\left|D_{i i}(A)\right| \gg\left|a_{11} a_{22} a_{33} a_{i i}^{-1}\right| \quad \forall A \in \Omega^{(i)} .
$$

Throughout this proof, the constants involved in the inequalities $\gg$ and in the notation $O(\cdot)$ depend on $\Omega$ but not on $N$.

Therefore, there is no loss of generality in assuming that

$$
\left|D_{33}(A)\right| \gg\left|a_{11} a_{22}\right| \quad \forall A \in \Omega .
$$

By Lemma 14, it suffices to show that

$$
\text { meas } P(N)=O\left(N^{3} \ln N\right) \text {, }
$$

where $P(N)=B(\partial \Omega([1 ; N]), 1)$. Put

$$
\begin{aligned}
K_{s}(N) & =\left\{x \in \mathbb{R}^{s}: 1 \leq x_{i}, i=1, \ldots, s, \prod_{i=1}^{s} x_{i} \leq N\right\} \quad \text { for } s \geq 2, \\
\underline{P}(N) & =\left\{A \in P(N): 1 \leq a_{i i}, i=1,2,3\right\} .
\end{aligned}
$$

Conditions (45) and (47) imply the relations

$$
\begin{aligned}
& a_{11} a_{22} a_{33}, a_{11} a_{22}, a_{11} a_{33}, a_{22} a_{33}, a_{i i} \in[0, c N], \quad c=c(\Omega), \\
& \left|a_{i j}\right| \ll a_{i i}+1, \quad i, j=1,2,3,
\end{aligned}
$$

for every $A \in P(N)$. If follows that

$$
\text { meas }(P(N) \backslash \underline{P}(N)) \ll 1+\int_{0}^{N}(x+1)^{2} d x+\int_{K_{2}(c N)}(x+1)^{2}(y+1)^{2} d x d y \ll N^{3} \ln N
$$

(the first summand is responsible for the case where all $a_{i i}$ are smaller than 1 , the second is responsible for the case where precisely two of the $a_{i i}$ are smaller than 1 , and the third for the case where precisely one $a_{i i}$ is smaller than 1$)$.

It suffices to estimate the measure of $\underline{P}(N)$. We denote by $\widetilde{P}(N)$ the set of $A \in \mathbb{R}^{3 \times 3}$ with

$$
\left|a_{i j}\right| \ll a_{i i}, i, j=1,2,3, \quad\left(a_{11}, a_{22}, a_{33}\right) \in K_{3}(c N) .
$$

Then $\underline{P}(N) \subset \widetilde{P}(N)$ by (49). By assumption, the set $\partial \Omega([1 ; N])$ is included in the union of a fixed number of $\widetilde{C}^{1}$-surfaces and $\mathrm{GL}_{3}(\mathbb{R} ; N), \mathrm{GL}_{3}(\mathbb{R} ; 1)$. Thus, it suffices to estimate the measures of the sets

$$
\begin{aligned}
& P_{1}(N)=\left\{A \in \widetilde{P}(N): \rho_{\infty}(A, L)<1\right\}, \\
& P_{2}(N)=\left\{A \in \widetilde{P}(N): \rho_{\infty}\left(A, \mathrm{GL}_{3}(\mathbb{R} ; N)\right)<1\right\}, \\
& P_{3}(N)=\left\{A \in \widetilde{P}(N): \rho_{\infty}\left(A, \mathrm{GL}_{3}(\mathbb{R} ; 1)\right)<1\right\},
\end{aligned}
$$

where the surface $L \in \widetilde{C}^{1}$ is independent of $N$.

We estimate $P_{1}(N)$. Let $L$ be defined by the equation $a_{k l}=f\left(A^{\prime}\right)$, where $A^{\prime}$ is the collection obtained by deleting the variable $a_{k l}$ from $\left(a_{11}, \ldots, a_{33}\right)$; the function $f \in C^{1}\left(\mathbb{R}^{8}\right)$ has uniformly bounded derivatives on $\mathbb{R}^{8}$. Without loss of generality, we assume that $k=3$. Then it can easily be checked that the inequality $\rho_{\infty}(A, L)<1$ entails

$$
\left|a_{3 l}-f\left(A^{\prime}\right)\right| \ll 1
$$


From (50) we deduce that

$$
\begin{aligned}
\left|a_{3 j}\right| & \ll a_{33} \ll \frac{N}{a_{11} a_{11}}, \quad j=1,2,3 \\
& \Longrightarrow \text { meas } P_{1}(N) \ll \int_{K_{2}(N)} a_{11}^{2} a_{22}^{2}\left(\frac{N}{a_{11} a_{22}}\right)^{2} d a_{11} d a_{22}=O\left(N^{3} \ln N\right) .
\end{aligned}
$$

We estimate meas $P_{2}(N)$. Let $A \in P_{2}(N)$. Then there exists a point $B$ satisfying $\rho_{\infty}(A, B)<1, D(B)=N$. Expanding the function $B \rightarrow D(B)$ in the Taylor series near $A$, keeping in mind that the 4 th order derivatives vanish, and using (50), we obtain

Consequently, the transformation

$$
\begin{aligned}
|N-D(A)| & =|D(B)-D(A)| \ll \sum_{i, j=1}^{3}\left(\left|D_{i j}(A)\right|+\left|a_{i j}\right|\right)+1 \\
& \ll a_{22} a_{33}+a_{11} a_{33}+a_{11} a_{22} \ll a_{11} a_{22}+\frac{N}{a_{11}}+\frac{N}{a_{22}} .
\end{aligned}
$$

$$
b_{33}=N-D(A), b_{i j}=a_{i j}, i, j=1,2,3,(i, j) \neq(3,3),
$$

takes $P_{2}(N)$ into the set $\widetilde{P}_{2}(N)$ whose points $B$ satisfy

$$
\begin{aligned}
& \left(b_{11}, b_{22}\right) \in K_{2}(c N), \quad\left|b_{i j}\right| \ll b_{i i}, j \neq i, i=1,2, \\
& \left|b_{3 j}\right| \ll \frac{N}{b_{11} b_{22}}, j=2,3, \quad\left|b_{33}\right| \ll\left(b_{11} b_{22}+\frac{N}{b_{11}}+\frac{N}{b_{22}}\right) .
\end{aligned}
$$

For the absolute value of the Jacobian, we have $\left|D_{33}(a)\right|=\left|D_{33}(b)\right| \gg b_{11} b_{22}$. Therefore,

$$
\begin{aligned}
\text { meas } P_{2}(N) & =\int_{\widetilde{P}_{2}(N)} \frac{d b_{11} \cdots d b_{33}}{\left|D_{33}(b)\right|} \\
& \ll \int_{K_{2}(c N)} b_{11}^{2} b_{22}^{2} \frac{N^{2}}{b_{11}^{2} b_{22}^{2}} \frac{1}{b_{11} b_{22}}\left(b_{11} b_{22}+\frac{N}{b_{11}}+\frac{N}{b_{22}}\right) d b_{22} d b_{33} \\
& =O\left(N^{3} \ln N\right) .
\end{aligned}
$$

The measure of $P_{3}(N)$ is estimated similarly. We have proved that meas $P_{i}(N)=$ $O\left(N^{3} \ln N\right)$; consequently, (48) is fulfilled.

The function $\mu$ was defined in $\S 1$.

Theorem 2. Let $W_{i}$ be a finitely connected subset of $\mathbb{R}^{2}$ with piecewise smooth boundary $\partial W_{i}(i=1,2,3)$, and let

$$
\Omega=\left\{\left(\begin{array}{lll}
a_{11} & a_{12} & a_{13} \\
a_{21} & a_{22} & a_{23} \\
a_{31} & a_{32} & a_{33}
\end{array}\right) \in \mathrm{GL}_{3}(\mathbb{R}): \begin{array}{ll}
\left(a_{12}, a_{13}\right) \in W_{1} \cdot a_{11}, & a_{11} \in \mathbb{R}_{+} \\
\left(a_{21}, a_{22}\right) \in W_{2} \cdot a_{22}, & a_{23} \in \mathbb{R}_{+} \\
\left(a_{31}, a_{32}\right) \in W_{3} \cdot a_{33}, & a_{33} \in \mathbb{R}_{+}
\end{array}\right\} .
$$

Moreover, suppose that there exists a constant $C=C\left(W_{1}, W_{2}, W_{3}\right)$ with

$$
a_{11} a_{22} a_{33} \leq C \cdot \operatorname{det} A \quad \forall A \in \Omega .
$$

Then for $N>1$ we have

$$
\# \omega([1 ; N])=N^{3}\left(\frac{\mu(\Omega)}{6} \cdot \ln ^{2} N+O_{\Omega}(\ln N)\right) .
$$

Proof. It can easily be observed that $\Omega$ satisfies the assumptions of Lemma 15. Thus, it suffices to prove the relation

$$
\text { meas } \Omega([1 ; N])=\frac{\mu(\Omega)}{6} N^{3}\left(\ln ^{2} N+O(\ln N)\right) .
$$


For this, we change the variables in the integral

$$
\operatorname{meas} \Omega([1 ; N])=\int_{\Omega([1 ; N])} d a_{11} \cdots d a_{33},
$$

putting

$$
a_{i j}=b_{i j} \cdot a_{i i} \text { for } i \neq j, \quad a_{i i}=b_{i i}, \quad i=1,2,3 .
$$

Recall that $D(A)=\operatorname{det} A$. Since

$$
\frac{D(A)}{a_{11} a_{22} a_{33}}=D^{\prime}(B), \quad D^{\prime}(B)=\operatorname{det}\left(\begin{array}{ccc}
1 & b_{12} & b_{13} \\
b_{21} & 1 & b_{23} \\
b_{31} & b_{32} & 1
\end{array}\right)
$$

the set $\Omega([1 ; N])$ is taken to

$$
W([1 ; N])=\left\{\begin{array}{cl} 
& \left(b_{12}, b_{13}\right) \in W_{1}, \\
B \in \mathbb{R}^{3 \times 3}: & \left(b_{21}, b_{23}\right) \in W_{2}, \quad \frac{1}{D^{\prime}(B)} \leq b_{11} b_{22} b_{33} \leq \frac{N}{D^{\prime}(B)} \\
\left(b_{31}, b_{32}\right) \in W_{3},
\end{array}\right\} .
$$

We observe that $D^{\prime}(B) \asymp 1$ for $B \in W([1 ; N]$ ) (this follows from the assumptions of the theorem). Integrating with respect to $b_{i i}$, we obtain

$$
\begin{aligned}
\operatorname{meas} & \Omega([1 ; N])=\int_{W([1 ; N])} b_{11}^{2} b_{22}^{2} b_{33}^{2} d b_{11} \cdots d b_{33} \\
& =\int_{W_{1} \times W_{2} \times W_{3}} \frac{N^{3}}{6\left(D^{\prime}(B)\right)^{3}}\left(\ln ^{2} N+O(\ln N)\right) d b_{12} d b_{13} d b_{21} d b_{23} d b_{31} d b_{32} \\
& =\frac{N^{6}}{6}\left(\ln ^{2} N+O(\ln N)\right) \cdot \mu(\Omega) .
\end{aligned}
$$

\section{§5. Proof of Theorem 1}

Put

$$
\omega_{i}([1, N])=\bigcup_{n=1}^{N} \omega_{i}(n), \quad \partial \omega_{i}([1, N])=\bigcup_{n=1}^{N} \partial \omega_{i}(n), \quad i=1,2,3,4 .
$$

The sets $\Omega_{i}$ were defined in $\S 1$. Since $\Omega_{i}$ and $\partial \Omega_{i}$ satisfy the assumptions of Theorem 2 , we have

$$
\# \omega_{i}([1, N])=\frac{\mu\left(\Omega_{i}\right)}{6} N^{3} \ln ^{2} N+O\left(N^{3} \ln N\right), \quad \# \partial \omega_{i}([1, N])=O\left(N^{3} \ln N\right) .
$$

The claim of Theorem 1 follows from this combined with (14) and (16).

\section{REFERENCES}

[1] F. Klein, Uber eine geometrische Auffassung der gewohlichen Kettenbruchentwichlung, Nachr. Ges. Wiss. Göttingen 1895, no. 3, 357-359.

[2] G. F. Voronor, Collected works in three volumes, Akad. Nauk USSR, Kiev, 1952. (Russian) MR0062686(16:2d)

[3] H. Minkowski, Généralisation de la théorie des fractions continues, Ann. Sci. École Norm. Sup. (3) 13 (1896), 41-60. MR.1508923

[4] V. A. Bykovskiı̌, On the error of number-theoretic quadrature formulas, Dokl. Akad. Nauk 389 (2003), no. 2, 154-155. (Russian) MR2003229

[5] _ On the error in number-theoretic quadrature formulas, Chebyshevskiŭ Sb. 3 (2002), no. 2, 27-33. (Russian) MR2035611(2005d:11102)

[6] N. M. Korobov, Number-theoretic methods in approximate analysis, 2nd ed., MTsNMO, Moscow, 2004. (Russian) MR2078157(2005f:41001)

[7] O. A. Gorkusha and N. M. Dobrovol'skiı̌, On estimates for the hyperbolic zeta function of lattices, Chebyshevskiŭ Sb. 6 (2005), no. 2, 129-137. (Russian) MR2262602 (2007j:11118) 
[8] A. A. Illarionov, An estimate for the number of relative minima of arbitrary-rank incomplete integer lattices, Dokl. Akad. Nauk 418 (2008), no. 8, 155-158; English transl., Dokl. Math. 77 (2008), no. 1, 31-34. MR2462063

[9] H. Heilbronn, On the average length of a class of finite continued fractions, Number Theory and Analysis, Papers in Honor of Edmund Landau, Plenum, New York, 1969, pp. 87-96. MR0258760 $(41: 3406)$

[10] M. O. Avdeeva, Lower bounds for the number of local minima of integer lattices, Fundam. Prikl. Mat. 11 (2005), no. 6, 9-14; English transl., J. Math. Sci. (N. Y.) 146 (2007), no. 2, 5629-5633. MR.2204418 (2006k:11127)

[11] G. Lochs, Statistik der Teilnenner der zu den echten Brüchen gehörigen regelmässigen Kettenbrüche., Monatsh. Math. 65 (1961), 27-52. MR0124308(23:A1622)

[12] J. W. Porter, On a theorem of Heilbronn, Mathematika 22 (1975), no. 1, 20-28. MR0498452 $(58: 16567)$

[13] D. E. Knuth, The art of computer programming. Vol. 2, Addison-Wesley Publ. Co., Reading, MA, 1981. MR0633878 (83i:68003)

[14] H. Minkowski, Zur Theorie der Kettenbrüche, Gesammelte Abhandlungen. Bd. I, Teubner, LeipzigBerlin, 1911, S. 278-292.

[15] V. A. Bykovskiı̌ and O. A. Gorkusha, Minimal bases of three-dimensional complete lattices, Mat. Sb. 192 (2001), no. 2, 57-66; English transl., Sb. Math. 192 (2001), no. 1-2, 215-223. MR1835985 (2002d:11075)

[16] O. A. Gorkusha, Minimal bases of three-dimensional complete lattices, Mat. Zametki 69 (2001), no. 3, 353-362; English transl., Math. Notes 69 (2001), no. 3-4, 320-328. MR1846834(2002d:11077)

[17] J. W. S. Cassels, An introduction to the geometry of numbers, Springer-Verlag, Berlin, 1997. MR 1434478 (97i:11074)

[18] A. V. Ustinov, Solution of the Arnol'd problem on weak asymptotics for Frobenius numbers with three arguments, Mat. Sb. 200 (2009), no. 4, 131-160; English transl., Sb. Math. 200 (2009), no. 3-4, 597-627. MR2531884 (2010e:11091)

Khabarovsk Division, Institute of Applied Mathematics, Russian Academy of Sciences, 54 Dzerzhinskiľ Street, Khabarovsk 680000, Russia

E-mail address: illar_a@list.ru

Received 30/NOV/2009

Translated by S. KISLYAKOV 